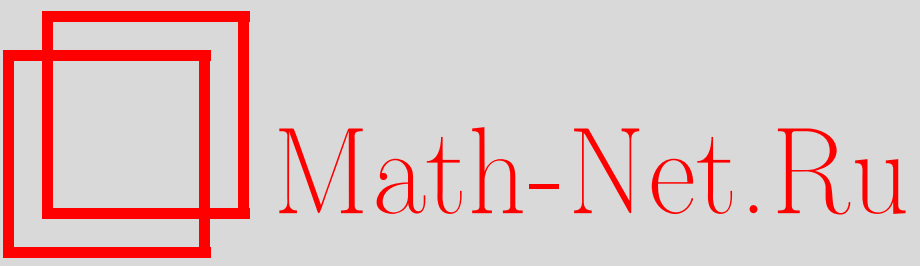

А. А. Туганбаев, Модули над наследственными нётеровыми первичными кольцами, УМH, 2000, том 55, выпуск 2, 151-152

DOI: https://doi.org/10.4213/rm283

Использование Общероссийского математического портала Math-Net.Ru подразумевает, что вы прочитали и согласны с пользовательским соглашением

http://www.mathnet.ru/rus/agreement

Параметры загрузки:

IP: 54.172 .240 .79

26 апреля 2023 г., 05:43:58 


\title{
МОДУЛИ НАД НАСЛЕДСТВЕННЫМИ НЕТЕРОВЫМИ ПЕРВИЧНЫМИ КОЛЬЦАМИ
}

\author{
А. А. ТУГАНБАЕВ
}

Модуль $M$ называется $\pi$-проективнылм, если для любых его подмодулей $U$ и $V$ таких, что $U+V=M$, сушествует такой эндоморфизм $f$ модуля $M$, что $f(M) \subseteq U$ и $(1-f)(M) \subseteq V$.

Лемма $1[1 ; 55.16,53.6]$. Если $A$ - полуцепное артиново кольцо, то $A$ - кольцо конечного типа представлений, и каждый $A$-модуль является прямой суммой циклических цепнъц квазипроективных модулей.

Лемма 2. Пусть А-наследственное нётерово первичное кольцо.

(1) Все собственные фактор-кольца кольца $А$ являются полуцепными артиновыми.

(2) Все А-модули без кручения являются плоскими.

(3) $A$ - либо ограниченное, либо примитивное кольцо. (Если $A$ - ограниченное примитивное кольцо, то $A$ - простое артиново кольцо.)

ДокАЗАТЕЛЬСТво. (1) доказано в [2; 25.5.1]. (2) доказано в [2; 2.4.7]. (3) доказано в [3].

Теорема 1. Пусть $M-м$ модуль над наследственным нётеровым первичным кольцом $A, X$ - чистый неточный подмодуль модуля $M$. Тогда $X$ - прямое слагаемое модуля $M$.

ДоказАтельство. Пусть $B \equiv r(X), R \equiv A / B, h: M \rightarrow M B$ - естественный эпиморфизм. Можно считать, что $0 \neq B \neq A$. Так как $X$ - чистый подмодуль в $M$, то $X \cap M B=$ $X B=0$. По леммам $2(1)$ и $1 R$ - кольцо конечного типа представлений. Поэтому все $R$-модули чисто-инъективны $[1 ; 54.3]$ и $h(M)$ - чисто-инъективный $R$-модуль.

Допустим, что $h(X)$ - чистый подмодуль чисто-инъективного $R$-модуля $h(M)$. Тогда $h(X)$ - прямое слагаемое $R$-модуля $h(M)$. Пусть $h(M)=h(X) \oplus h(Y)$, где $M B \subseteq Y \subseteq M$. Тогда $M=X+Y$ и $X \cap Y=X \cap M B=0$. Поэтому $M=X \oplus Y$.

Остается доказать, что $h(X)$ - чистый подмодуль $R$-модуля $h(M)$. Пусть $\bar{D} \equiv D / B$ - любой левый идеал кольца $R$, где $D$ - левый идеал кольца $A$, содержащий $B$. Пусть $h(x) \in h(X) \cap$ $h(M) \bar{D}$, где $x \in X$. Тогда существуют такие элементы $d_{1}, \ldots, d_{k} \in D, b_{1}, \ldots, b_{n} \in B$ и $m_{1}, \ldots, m_{k}, t_{1}, \ldots, t_{n} \in M$, что $x=\sum_{i=1}^{k} m_{i} d_{i}+\sum_{j=1}^{n} t_{j} b_{j} \in M(D+B) \cap X$. Так как $X$ - чистый подмодуль в $M$, то $M(D+B) \cap X=X(D+B)$. Поэтому $h(x) \in h(X D)+h(X B)=h(X) \bar{D}$. Тогда $h(X) \cap h(M) \bar{D} \subseteq h(X) \bar{D}$ и $h(X)$ - чистый подмодуль $A / B$-модуля $h(M)$.

ПреДложениЕ 1. Пусть $A$ - ограниченное наследственное нётерово первичное кольчо, $M-A$-модуль с ненулевой периодической частью $T$. Тогда $M$ имеет ненулевое чепное счетно порожденное периодическое прямое слагаемое $X$, которое может быть въбрано ииклическим неточным модулем в случае неиндективности модуля Т. Следовательно, если модуль $T$ не индективен, то $M$ имеет ненулевое чиклическое чепное неточное прямое слагаемое.

ДокАЗАТЕльство. Так как $M / T-A$-модуль без кручения, то $M / T$ плосок по лемме $2(2)$. Поэтому $T$ чист в $M$ [4; с. 37]. Можно считать, что $A$ не является простым артиновым. По лемме 2 (3) $A$ не является примитивным справа. По теореме 10 из [5] $T$ имеет ненулевое равномерное периодическое прямое слагаемое $X$, которое может быть выбрано циклическим неточным модулем в случае неинъективности модуля $T$. Так как $X$ - прямое слагаемое чистого подмодуля $T$ в $M$, то $X$ - чистый подмодуль в $M$. Пусть $E$ - инъективная оболочка равномерного модуля $X$. По теореме 4 из [6] $E$ - цепной счетно порожденный модуль, у которого все собственные подмодули цикличны. Тогда $X$ - цепной модуль и либо $X=E$, либо $X$ цикличен. Поэтому достаточно доказать, что $X$ - прямое слагаемое в $M$. Это так, если $X$ инъективен. Допустим, что $X$ цикличен. По лемме 2 из [6] $r(X) \neq 0$. По теореме $1 X$-прямое слагаемое в $M$.

Работа выполнена при поддержке Российского фонда фундаментальных исследований (грант № 99-01-00382). 
Лемма 3. Пусть $M$ и $Y_{i}(i \in I)-A$-модули.

(1) Если все $Y_{i}$ M-проективнь, то $\bigoplus_{i \in I} Y_{i} M$-проективен.

(2) Если $Y-$ подфактор модуля $\bigoplus_{i \in I} Y_{i}, X-м$ модуль, проективный относительно всех модулей $Y_{i}$, причем либо $I$ конечно, либо $X$ конечно порожден, то $X Y$-проективен.

(3) Если $\bigoplus_{i \in I} Y_{i}$ л-проективен, то $Y_{i} Y_{j}$-проективен для любъх несовпадающих $i, j \in I$.

(4) Если А артиново справа, то $M_{A}$ квазипроективен $\Longleftrightarrow M_{A / r(M)}$ проективен.

(5) Если $M$ квазипроективен, то $M \pi$-проективен.

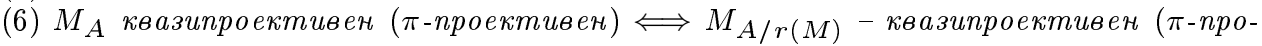
ективен).

ДокАЗАтЕльство. (1), (2) и (3) доказаны в $[1 ; 18.1,18.2,41.14]$. (4) доказано в теореме 2.3 из [7]. (5) см. в [1; с. 369]. (6) проверяется непосредственно.

ЛЕмма 4. Пусть модуль $M$ является прямой суммой конечно порожденных модулей $M_{i}(i \in I)$. Равносильны условия:

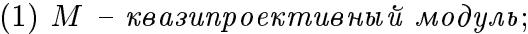

(2) $M \pi$-проективен и все $M_{i}$ квазипроективны;

(3) $M_{i} M_{j}$-проективен для всех $i, j \in I$.

ДокАЗАТЕльСтво. (1) $\Longrightarrow(2)$ следует из леммы $3(5)$ и того, что прямые слагаемые квазипроективных модулей квазипроективны. $(2) \Longrightarrow(3)$ следует из квазипроективности модулей $M_{i}$ и того, что по лемме $3(3) \quad M_{i} \quad M_{j}$-проективен для любых несовпадающих $i, j \in I .(3) \Longrightarrow(1)$ следует из лемм $3(1)$ и $3(2)$.

Лемма 5. (1) Пусть $M$ - прямая сумма конечно порожденных квазипроективных модулей. Тогда $M$ квазипроективен $\Longleftrightarrow M \pi$-проективен.

(2) Если $X \oplus N$ л-проективен и $Y$ - подфактор модуля $N$, то $X X$-проективен.

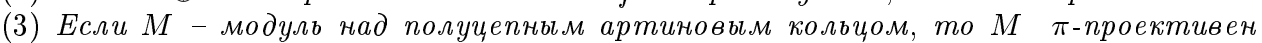
$\Longleftrightarrow M$ квазипроективен $\Longleftrightarrow M-$ проективныи $A / r(M)$-модуль.

ДокАЗАТЕЛЬСтво. (1) следует из леммы 4. (2) следует из лемм 3 (3) и 3 (2). (4) следует из (1), (2) и лемм $3(4)$ и 4.

ПРЕДЛОЖенИЕ 2. Если $M$ - неточный модуль над наследственнылм нётеровым первичньм кольцом $A$, то $M_{A} \pi$-проективен $\Longleftrightarrow M_{A}$ квазипроективен $\Longleftrightarrow M_{A / r(M)}$ проективен.

ДокАЗАТЕльство. Предложение 2 следует из лемм $2(1), 3(6), 5(3)$.

\section{СПИСОК ЛИТЕРАТУРЫ}

[1] Wisbauer R. Foundations of Module and Ring Theory. Philadelphia: Gordon and Breach, 1991. [2] Фейс К. Алгебра: кольца, модули и категории. Т. 2. М.: Мир, 1979. [3] Lenagan Т. Н // J. London Math. Soc. 1973. V. 6. P. 241-246. [4] Stenström B. Rings of Quotients: An Introduction to Methods of Ring Theory. Berlin: Springer-Verlag, 1975. [5] Singh S. // Canad. J. Math. 1975. V. 27. № 4. P. 867-883. [6] Singh S. // Canad. J. Math. 1974. V. 26. № 5. P. 1173-1185. [7] Fuller K. R., Hill D. A. // Arch. Math. 1970. V. 21. P. 369-373. 